PROCEEDINGS OF THE

AMERICAN MATHEMATICAL SOCIETY

Volume 35, Number 2, October 1972

\title{
A COBORDISM
}

\author{
R. E. STONG
}

\begin{abstract}
A cobordism of complex projective $n$-space and the square of real projective $n$-space is exhibited.
\end{abstract}

1. Introduction. The object of this note is to prove

Proposition. Complex projective n-space, $C P(n)$, is cobordant to the square of real projective $n$-space, $R P(n) \times R P(n)$.

This result was announced by Rohlin [4] in 1958 and proved by Wall in $[5$, p. 300], who noted that these manifolds have the same Stiefel-Whitney numbers. A geometric argument was given by Conner and Floyd [2, p. 63] by analyzing involutions, and their methods may be used to construct an explicit cobordism.

Aesthetically, one would like to write down a manifold whose boundary is the disjoint union of $C P(n)$ and $R P(n) \times R P(n)$.

Let $C P(n)$ be written as the set of equivalence classes of $(n+1)$-tuples $\left[z_{0}, \cdots, z_{n}\right]$ of complex numbers with $z_{0} \bar{z}_{0}+\cdots+z_{n} \bar{z}_{n}=1$, where $\left[\lambda z_{0}\right.$, $\left.\cdots, \lambda z_{n}\right]=\left[z_{0}, \cdots, z_{n}\right]$ if $\lambda$ is a complex number with norm $|\lambda|=1$, where $\bar{z}$ is the conjugate of $z$.

Let $W$ be the space obtained from $C P(n) \times[0,1]$ by identifying $\left(\left[z_{0}\right.\right.$, $\left.\left.\cdots, z_{n}\right], 1\right)$ with $\left(\left[\bar{z}_{0}, \cdots, \bar{z}_{n}\right], 1\right)$ if $\left|\left(z_{0}^{2}+\cdots+z_{n}^{2}\right)\right| \leqq 3 / 5$.

Claim. $W$ is a manifold with boundary and the boundary of $W$ is the disjoint union of $C P(n)$ and $R P(n) \times R P(n)$.

2. The proof. To begin the proof, let $r: C P(n) \rightarrow R$ be the real-valued function defined by $r\left(\left[z_{0}, \cdots, z_{n}\right]\right)=\left|\left(z_{0}^{2}+\cdots+z_{n}^{2}\right)\right|$. Since $\mid\left(z_{0}^{2}+\cdots+\right.$ $\left.z_{n}^{2}\right)|\leqq| z_{0}^{2}|+\cdots+| z_{n}^{2} \mid=1$, this function takes values in the interval $[0,1]$.

For any $(n+1)$-tuple $\left(z_{0}, \cdots, z_{n}\right)$ of complex numbers with $z_{0} \bar{z}_{0}+$ $\cdots+z_{n} \bar{z}_{n}=1$, one has $z_{0}^{2}+\cdots+z_{n}^{2}=r\left(\left[z_{0}, \cdots, z_{n}\right]\right) e^{i \theta}$ for some $\theta$. If $r\left(\left[z_{0}, \cdots, z_{n}\right]\right)>0$, there are exactly two $(n+1)$-tuples $\left(u_{0}, \cdots, u_{n}\right)$ and $\left(-u_{0}, \cdots,-u_{n}\right)$ for which $\left[u_{0}, \cdots, u_{n}\right]=\left[z_{0}, \cdots, z_{n}\right]$ and with $u_{0}^{2}+$ $\cdots+u_{n}^{2}=r\left(\left[z_{0}, \cdots, z_{n}\right]\right)$, given by $u_{j}=z_{j} e^{-i \theta / 2}$. If $u_{j}=x_{j}+i y_{j}$, then

Received by the editors December 29, 1971 .

AMS 1970 subject classifications. Primary 57D75.

${ }^{1}$ I am indebted to the National Science Foundation for financial support during this work.

c. American Mathematical Society 1972 
$u_{0}^{2}+\cdots+u_{n}^{2}=\left(\|x\|^{2}-\|y\|^{2}\right)+2 i x \cdot y, \quad$ where $\quad x=\left(x_{0}, \cdots, x_{n}\right), \quad y=\left(y_{0}\right.$, $\left.\cdots, y_{n}\right),\|x\|^{2}=x_{0}^{2}+\cdots+x_{n}^{2}$ and $x \cdot y=x_{0} y_{0}+\cdots+x_{n} y_{n}$, and so $u_{0}^{2}+$ $\cdots+u_{n}^{2}$ is a positive real if and only if $\|x\|^{2}-\|y\|^{2}>0$ and $x \cdot y=0$.

Let $V=\left\{(x, y) \in R^{n+1} \times R^{n+1} \mid\|x\|=1,\|y\|<1, x \cdot y=0\right\}$ and let $\varphi: V \rightarrow$ $C P(n)$ by

$$
\varphi\left(\left(x_{0}, \cdots, x_{n}\right),\left(y_{0}, \cdots, y_{n}\right)\right)=\left[\left(x_{0}+i y_{0}\right) / t, \cdots,\left(x_{n}+i y_{n}\right) / t\right]
$$

where $t=\left(1+\|y\|^{2}\right)^{1 / 2}$. If $V^{\prime}$ is the quotient space of $V$ obtained by identifying $(x, y)$ with $(-x,-y)$, then $\varphi$ induces a diffeomorphism $\varphi^{\prime}: V^{\prime} \rightarrow$ $C P(n)$ onto the open set of $C P(n)$ consisting of points $\left[z_{0}, \cdots, z_{n}\right]$ with $z_{0}^{2}+\cdots+z_{n}^{2} \neq 0$. Further $r \varphi^{\prime}(x, y)=\left(1-\|y\|^{2}\right) /\left(1+\|y\|^{2}\right)$. Thus $3 / 5$ is a regular value of the function $r: C P(n) \rightarrow R$, with $T=\left\{\left[z_{0}, \cdots, z_{n}\right] \in\right.$ $\left.C P(n) \mid r\left(\left[z_{0}, \cdots, z_{n}\right]\right) \leqq 3 / 5\right\}$ being a regularly imbedded submanifold of $C P(n)$ on which the involution sending $\left[z_{0}, \cdots, z_{n}\right]$ to $\left[\bar{z}_{0}, \cdots, \bar{z}_{n}\right]$ acts freely. Thus the space $W$ is a differentiable manifold with boundary.

One boundary component of $W$ is $C P(n)$, given by $C P(n) \times 0$ and the other, $M$, may be obtained from

$$
V^{\prime \prime}=\left\{(x, y) \in R^{n+1} \times R^{n+1} \mid\|x\|=1,\|y\| \leqq \frac{1}{2}, x \cdot y=0\right\}
$$

by identifying $(x, y)$ and $(-x,-y)$ and by identifying $(x, y)$ with $(x,-y)$ if $\|y\|=\frac{1}{2}$, this being the identification of $\left[z_{0}, \cdots, z_{n}\right]$ with $\left[\bar{z}_{0}, \cdots, \bar{z}_{n}\right]$ where $r\left(\left[z_{0}, \cdots, z_{n}\right]\right)=3 / 5$.

Now let $M^{\prime}$ be the manifold obtained from

$$
U=\left\{(x, y, t) \in R^{n+1} \times R^{n+1} \times R \mid\|x\|=1,\|y\|^{2}+t^{2}=1, x \cdot y=0\right\}
$$

by identifying $(x, y, t)$ with $(-x,-y, t)$ and $(x, y, t)$ with $(x,-y,-t)$. Then $\psi: V^{\prime \prime} \rightarrow U$ defined by $\psi((x, y))=\left(x, 2 y,\left(1-4\|y\|^{2}\right)^{1 / 2}\right)$ maps $V^{\prime \prime}$ onto the subset with $t \geqq 0$ and induces a diffeomorphism of $M$ with $M^{\prime}$.

Now let $M^{\prime \prime}$ be the manifold obtained from

$$
U^{\prime}=\left\{(x, y) \in R^{n+1} \times R^{n+1} \mid\|x\|=1,\|y\|=1\right\}
$$

by identifying $(x, y)$ with $(-x,-y)$ and $(x, y)$ with $(x,-y)$. Then $\eta: U \rightarrow U^{\prime}$ defined by $\eta(x, y, t)=(x, y+t x)$ induces a diffeomorphism of $M^{\prime}$ with $M^{\prime \prime}$.

However, the identification of $(x, y)$ with $(-x,-y)$ and $(x,-y)$ on $U^{\prime}$ is the same as the identification of $(x, y)$ with $(-x, y)$ and $(x,-y)$, for these involutions generate the same $Z_{2} \times Z_{2}$ action. Thus $M^{\prime \prime}$ is $R P(n) \times$ $R P(n)$, which completes the proof.

Note. In this proof, explicit maps and manifolds have been described at each step. This has tended to obscure the logic of the argument, which is as follows. Following Landweber [3], one has chosen an explicit tubular neighborhood $\varphi\left(V^{\prime \prime}\right)$ of $R P(n)$ in $C P(n)$ invariant under conjugation. Since 
the normal bundle of $R P(n)$ in $C P(n)$ is the tangent bundle $\tau$ of $R P(n)$, this is just a copy of the disc bundle $D(\tau)$. The manifold $M$ is then obtained by identifying antipodal points of the sphere bundle $S(\tau)$. This identification space is just the real projective space bundle of $\tau \oplus \mathbf{1}$, where 1 is a trivial line bundle, and $M^{\prime}=R P(\tau \oplus \mathbf{1})$. The manifold $M^{\prime \prime}$ is just the real projective space bundle $R P((n+1) \lambda)$ where $\lambda$ is the canonical line bundle over $R P(n)$, with $\eta$ being obtained from the isomorphism of $\tau \oplus 1$ with $(n+1) \lambda$. Finally, as noted by Atiyah [1, p. 45], if $\lambda$ is a line bundle $R P(\xi \otimes \lambda)$ is the same as $R P(\xi)$, so $R P((n+1) \lambda)=R P((n+1))$, where $(n+1)$ is the trivial $(n+1)$-plane bundle, whose projective space is patently $R P(n) \times$ $R P(n)$, and this gives the observation that $M^{\prime \prime}$ is $R P(n) \times R P(n)$.

\section{REFERENCES}

1. M. F. Atiyah, $K$-theory, Benjamin, New York, 1967. MR 36 \#7130.

2. P. E. Conner and E. E. Floyd, Differentiable periodic maps, Ergebnisse der Mathematik und ihrer Grenzgebiete, N.F., Band 33, Academic Press, New York; SpringerVerlag, Berlin, 1964. MR 31 \#750.

3. P. S. Landweber, Fixed point free conjugations on complex manifolds, Ann. of Math. (2) 86 (1967), 491-502. MR 36 \#3382.

4. V. A. Rohlin, Internal homologies, Dokl. Akad. Nauk SSSR 119 (1958), 876-879. (Russian) MR 21 \#2238.

5. C. T. C. Wall, Determination of the cobordism ring, Ann. of Math. (2) 72 (1960), 292-311. MR 22 \#11403.

Department of Mathematics, University of Virginia, Charlottesville, Virginia 22904 